\title{
Differences in Prevalence of Lymphovascular Invasion among Early Gastric Cancers between Korea and Japan
}

Sun-Young Lee ${ }^{1}$, Naohisa Yoshida ${ }^{2}$, Osamu Dohi ${ }^{2}$, Sang Pyo Lee ${ }^{1}$, Daisuke Ichikawa ${ }^{3}$, Jeong Hwan Kim ${ }^{1}$, In-Kyung Sung ${ }^{1}$, Hyung Seok Park ${ }^{1}$, Eigo Otsuji ${ }^{3}$, Yoshito Itoh ${ }^{2}$, Chan Sup Shim ${ }^{1}$, Hye Seung Han $^{4}$, Mitsuo Kishimoto ${ }^{5}$, and Yuji Naito ${ }^{2}$

${ }^{\prime}$ Department of Internal Medicine, Konkuk University School of Medicine, Seoul, Korea, Departments of ${ }^{2}$ Molecular Gastroenterology and Hepatology and ${ }^{3}$ Surgery, Kyoto Prefectural University of Medicine, Kyoto, Japan, ${ }^{4}$ Department of Pathology, Konkuk University School of Medicine, Seoul, Korea, and ${ }^{5}$ Department of Surgical Pathology, Kyoto Prefectural University of Medicine, Kyoto, Japan

Background/Aims: The presence of invasion is a diagnostic criterion of early gastric cancer (EGC) in Korea, whereas diagnosis in Japan is based on enlarged nuclei and prominent nucleoli. Moreover, the depth of invasion is the location of cancer cell infiltration in Korea, whereas it is the location of lymphovascular invasion (LVI) or cancer cell infiltration in Japan. We evaluated the characteristics of EGC with LVI to uncover the effects of different diagnostic criteria. Methods: Consecutive T1-stage EGC patients who underwent complete resection were included after endoscopic or surgical resection. The presence of LVI was evaluated. Results: LVI was present in 112 of 1,089 T1-stage EGC patients. LVI was associated with depth of invasion $(p<0.001)$ and age $(p=0.017)$. The prevalence of $\mathrm{LVI}$ in mucosal cancer was significantly higher in Korea $(p<0.001)$, whereas that of submucosal cancer was higher in Japan $(p=0.024)$. For mucosal EGC types, LVI was positively correlated with diagnostic criteria applied in Korea $(p=0.017)$. For submucosal EGC types, LVI was positively correlated with Japanese criteria $(p=0.001)$ and old age $(p=0.045)$. Conclusions: The higher prevalence of LVI for mucosal EGC in Korea and for submucosal EGC in Japan indicates that different diagnostic criteria should be considered when reading publications from other countries.

(Gut Liver 2017;11:383-391)

Key Words: Stomach neoplasms; Early gastric cancer; Invasion; Depth

\section{INTRODUCTION}

The use of endoscopic resection is spreading rapidly in countries with a high prevalence of gastric neoplasms. Nonetheless, the prevalence of adenoma and early gastric cancer (EGC) differ between countries because of differences in the applied pathologic criteria. ${ }^{1,2}$ For example, structural invasion is necessary to diagnose EGC outside of Japan, while severe cytologic atypia with enlarged oval nuclei and prominent nucleoli is diagnosed as EGC in Japan regardless of the presence of invasion. ${ }^{3-5}$ Therefore, lesions that most pathologists in Korea identify as dysplasia are often considered to be intramucosal cancer in Japan (Fig. 1).

Besides this inconsistency in the diagnosis of intramucosal cancer and dysplasia, different criteria are applied to determine the final depth of invasion of EGC between countries. ${ }^{6-8}$ The

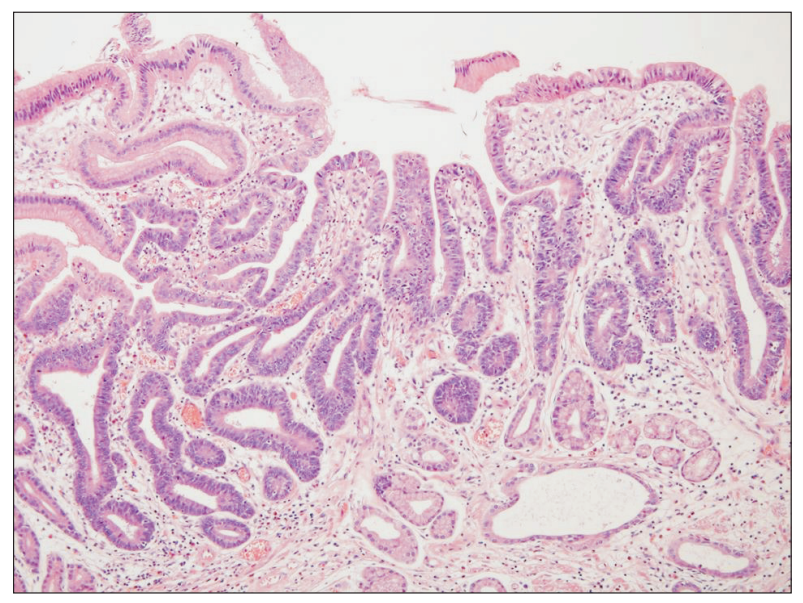

Fig. 1. A Korean case of gastric adenoma with high-grade dysplasia that is regarded as early gastric cancer (EGC) in Japan. Because dysplastic cytologic atypia is confined to the superficial mucosa without invasion into the lamina propria, the diagnosis in Korea is not cancer but a gastric adenoma with high-grade dysplasia (H\&E stain, $\times 100$ ). The diagnosis is EGC in Japan based on severe cytologic atypia with enlarged nuclei.

\section{Correspondence to: Sun-Young Lee}

Department of Internal Medicine, Konkuk University School of Medicine, 120-1 Neungdong-ro, Gwangjin-gu, Seoul 05030, Korea

Tel: +82-2-2030-7747, Fax: +82-2-2030-7748, E-mail: sunyoung@kuh.ac.kr

Received on June 6, 2016. Revised on July 16, 2016. Accepted on August 21, 2016. Published online January 20, 2017

pISSN 1976-2283 eISSN 2005-1212 https://doi.org/10.5009/gnl16281

() This is an Open Access article distributed under the terms of the Creative Commons Attribution Non-Commercial License (http://creativecommons.org/licenses/by-nc/4.0) which permits unrestricted non-commercial use, distribution, and reproduction in any medium, provided the original work is properly cited. 


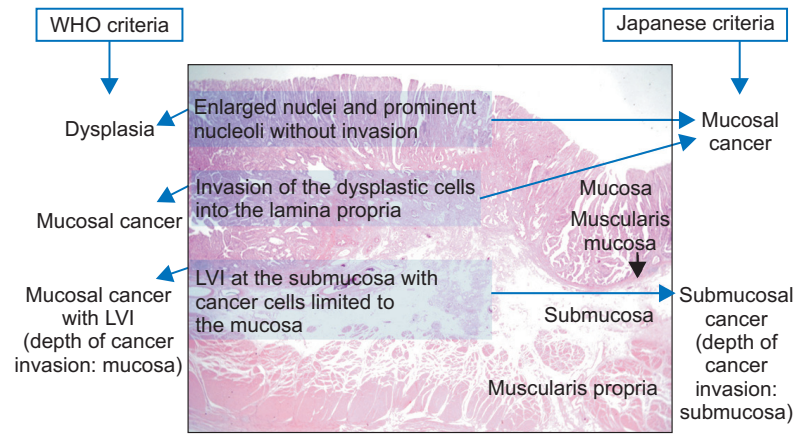

Fig. 2. Different diagnostic criteria between the two countries. A diagnosis of early gastric cancer (EGC) is based on the presence of cancer cell invasion into the lamina propria in Korea, whereas it is based on severe dysplastic atypia with enlarged vesicular oval nuclei and prominent nucleoli, irrespective of the presence of invasion, in Japan. The final depth of cancer invasion is the location of lymphovascular invasion (LVI) in Japan, whereas it is the location of cancer cells in Korea. For these reasons, EGC cases are usually graded higher in Japan than in Korea.

WHO, World Health Organization.

final depth of cancer invasion is the location of cancer cell infiltration regardless of lymphovascular invasion (LVI) in Korea according to the World Health Organization (WHO) criteria, whereas it is the location of LVI or cancer cell infiltration in Japan. Therefore, when the cancer cells are located within the mucosal layer and LVI is present in the submucosal layer, the condition is diagnosed as a mucosal EGC with LVI in Korea, but as submucosal EGC in Japan (Fig. 2). Due to the differences in the definitions of cancer and depth of invasion, gastric neoplasms are usually of higher grade in Japan than in Korea.

Endoscopic resection for EGC is widely performed, and LVI is an important factor for incomplete resection, recurrence and poor prognosis after resection. ${ }^{9-11}$ Recent studies showed that LVI and depth of invasion are independent risk factors for lymph node (LN) metastasis in EGC. ${ }^{12-15}$ Based on these findings, additional surgery is recommended after endoscopic resection for EGCs with LVI. Therefore, LVI status should be always considered when resecting EGC endoscopically. ${ }^{16}$

The aim of the present study was to identify the characteristics of EGCs with LVI with the aim of uncovering whether the application of different diagnostic criteria affects the reported prevalence of LVI. In detail, we evaluated the characteristics of EGCs with LVI, and further analyzed whether the prevalence of LVI in T1-stage EGCs differs between Japan and Korea.

\section{MATERIALS AND METHODS}

\section{EGCs after complete resection}

Consecutive T1-stage EGC patients who underwent complete resection either endoscopically or surgically between 2010 and 2014 at Kyoto Prefectural University of Medicine $(n=776)$ and Konkuk University Medical Center $(n=313)$ were included in this cross-sectional study. EGCs other than T1a or T1b stage (i.e., Tis-stage EGC), incomplete resection, metachronous EGCs, and synchronous EGCs were excluded. EGCs that revealed either LN enlargement on computed tomography (CT) scan or LVI after endoscopic resection were also classified as incomplete resection in this study. Furthermore, cancer cells other than adenocarcinoma such as gastric lymphoma and gastrointestinal stromal tumor were excluded.

This cross-sectional study was approved by the Institutional Review Board (IRB) of Kyoto Prefectural University (IRB approval number: ERB-C-586) and Konkuk University (IRB approval number: KUH1010644). The study was registered at the Clinical Trial Registration sites in Japan (http://www.umin.ac.jp/ english/) as UMIN000019935 and in Korea (http://cris.nih.go.kr) as KCT0001490.

\section{Cell types of EGC}

All cases were analyzed by the pathologists at each university according to their pathologic criteria. The definition of EGC was severe cytologic dysplasia regardless of invasion in Japan, whereas it was dysplastic cells invading the lamina propria in Korea. Cell types were classified into well-differentiated (WD), moderately-differentiated (MD), poorly-differentiated (PD), mucinous adenocarcinoma, and poorly-cohesive carcinoma (including signet ring cell carcinoma) according to the dominant cell type of EGC.

\section{Presence of LVI}

Desmin staining was performed to determine the extent of submucosal invasion in both countries, in addition to hematoxylin \& eosin (HE) staining. Furthermore, immunohistochemistry (IHC) was performed using a primary antibody against ERG (Biocare Medical Inc., Concord, CA, USA) in Korea. ${ }^{17}$ In Japan, elastic-HE (EHE) stain was performed to evaluate the presence of venous invasion, and IHC stain for D2-40 was performed using a mouse monoclonal antibody against human lymphatic endothelium antigen (Nichirei, Tokyo, Japan) to evaluate lymphatic invasion. $^{18}$

\section{Final depth of cancer invasion}

Depth of invasion was taken as the level of cancer cell infiltration or LVI in Japan, whereas it was taken as the level of cancer cell infiltration regardless of the depth of LVI in Korea. $\mathrm{pN}$ staging was done after $\mathrm{LN}$ dissection for surgically resected cases. Numbers of LN metastases among the examined LN cases were recorded.

\section{Statistical analysis}

Analyses were conducted using PASW Statistics version 17.0 (SPSS Inc., Chicago, IL, USA), and significance was considered at $p$-value $<0.05$. Continuous variables are shown as mean value \pm standard deviation using Student t-test, while categori- 
cal variables are shown as frequency (\%) using the chi-square test. Variables with skewed distributions are shown as median value with ranges (minimum and maximum values) using the Kruskal-Wallis test. Logistic regression analysis was done to verify the independent significance of variables correlated with LVI using the odds ratio (OR) and 95\% confidence interval (CI).

\section{RESULTS}

\section{Characteristics of 1,089 EGCs}

The study involved 1,089 T1-stage EGCs, which included 112 cases (10.3\%) exhibiting LVI (Fig. 3). Endoscopic resection was applied to $473(61.0 \%)$ of the 776 T1-stage EGCs from Japan, and to $67(21.4 \%)$ of the 313 T1-stage EGCs from Korea. The overall LVI rate did not differ between the two countries (Table 1). Nonetheless, the LVI rate was significantly higher in Korea than in Japan for mucosal EGCs ( $p<0.001)$, whereas it was significantly higher in Japan than in Korea for submucosal cancers ( $\mathrm{p}=0.024)$.

\section{Variables related to LVI}

Differences in the characteristics of the 112 EGCs with LVI and of the 977 EGCs without LVI were analyzed. The age of the patient $(p=0.013)$, cell type $(p<0.001)$, depth of invasion $(p<0.001)$, pN staging $(p<0.001)$, and treatment $(p<0.001)$ differed according to the presence of LVI (Table 2). Logistic regression analyses revealed that a greater depth of cancer invasion was the most significant factor correlated with LVI, followed by old age (Table 3). EGCs with LVI were associated with a greater depth of invasion (OR of 0.023 for the mucosal layer, $\mathrm{p}<0.001$ ) and older age (OR, 1.027; $\mathrm{p}=0.017$ ).

\section{LVI in mucosal EGCs}

The patients were further subclassified into mucosal and submucosal EGC groups because the depth of invasion was the

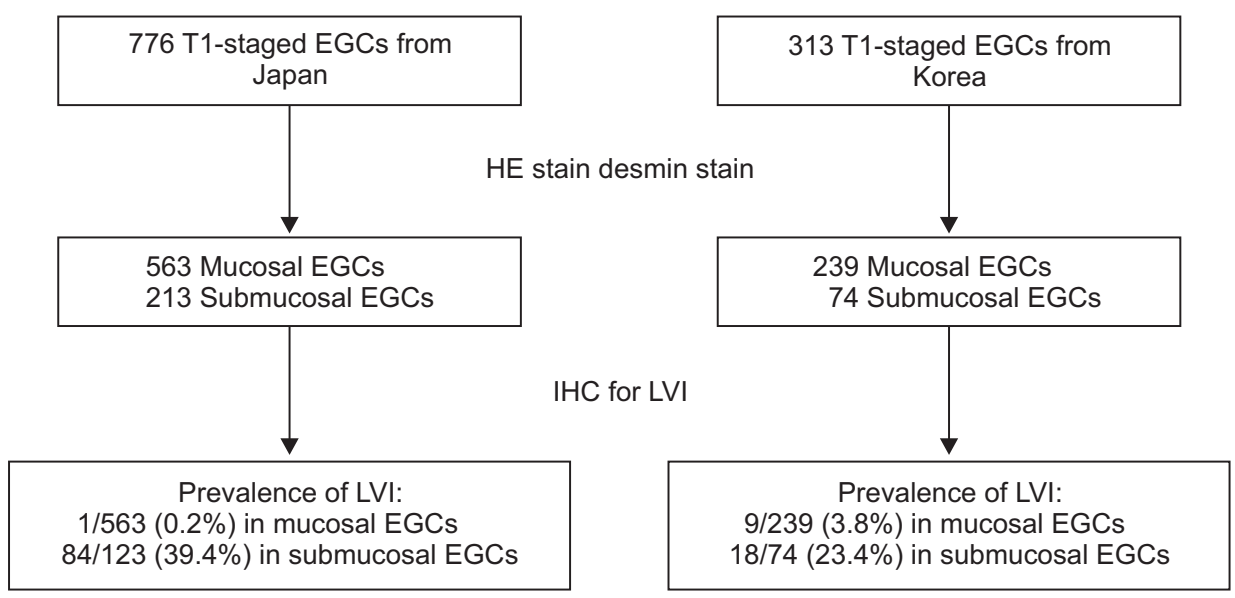

Fig. 3. Flow chart of the included subjects. For the evaluation of lymphovascular invasion (LVI), immunohistochemistry (IHC) staining for D2-40 and elastic-hematoxylin \& eosin (HE) were performed in Japan, whereas ERG IHC staining was performed in Korea. EGC, early gastric cancer.

Table 1. Baseline Characteristics of the 1,089 Early Gastric Cancer Patients

\begin{tabular}{|c|c|c|c|c|}
\hline \multirow{2}{*}{ Variable } & \multirow{2}{*}{ All $(n=1,089)$} & \multicolumn{3}{|c|}{ Differences between the two countries } \\
\hline & & Japan $(n=776)$ & Korea $(n=313)$ & p-value \\
\hline Age, yr & $66.0 \pm 11.1$ & $67.6 \pm 10.3$ & $61.9 \pm 12.0$ & $<0.001$ \\
\hline Male sex & $763(70.1)$ & $544(70.1)$ & $219(70.0)$ & 0.510 \\
\hline Size, $\mathrm{mm}$ & $19.7(1-145)$ & $17.8(1-145)$ & $24.6(2-114)$ & $<0.001$ \\
\hline Location (lower:middle:upper) & 430:491:168 & 283:339:154 & $147: 152: 14$ & $<0.001$ \\
\hline Cell type (WD:MD:PD:poorly-cohesive ${ }^{*}:$ mucinous type) & 575:236:96:176:6 & 519:102:30:120:5 & 56:134:66:56:1 & $<0.001$ \\
\hline Depth of invasion (mucosa) & $802(73.6)$ & $563(72.6)$ & $239(76.4)$ & 0.224 \\
\hline pN stage (NO:N1:N2:N3) & 1038:34:12:5 & $741: 24: 8: 3$ & $297: 10: 4: 2$ & 0.930 \\
\hline Treatment (endoscopic resection:surgical resection) & $540: 549$ & 473:303 & $67: 246$ & $<0.001$ \\
\hline Lymphovascular invasion & $112(10.3)$ & $85(11.0)$ & $27(8.6)$ & 0.272 \\
\hline Mucosal cancer & $10(1.3)$ & $1 / 563(0.2)$ & $9 / 239(3.8)$ & $<0.001$ \\
\hline Submucosal cancer & $102(35.5)$ & $84 / 213(39.4)$ & $18 / 74(24.3)$ & 0.024 \\
\hline
\end{tabular}

Data are presented as mean $\pm \mathrm{SD}$, number (\%), or median (range).

WD, well differentiated; MD, moderately differentiated; PD, poorly differentiated.

*Poorly cohesive carcinoma includes signet ring cell carcinoma. 
Table 2. Differences According to the Presence of Lymphovascular Invasion

\begin{tabular}{|c|c|c|c|}
\hline & With LVI & Without LVI & $\mathrm{p}$-value \\
\hline \multicolumn{4}{|l|}{ All EGCs $(\mathrm{n}=1,089)$} \\
\hline No. & 112 & 977 & - \\
\hline Age, yr & $68.5 \pm 9.7$ & $65.2 \pm 11.6$ & 0.013 \\
\hline Male sex & $76(67.9)$ & $687(70.3)$ & 0.587 \\
\hline Country (Japan:Korea) & $85: 27$ & 691:286 & 0.272 \\
\hline Size, $\mathrm{mm}$ & $30.3(5-132)$ & $28.9(6-119)$ & 0.241 \\
\hline Location (lower:middle:upper) & $40: 46: 26$ & 390:445:142 & 0.055 \\
\hline Cell type (WD:MD:PD:poorly-cohesive*:mucinous) & $38: 34: 13: 23: 4$ & 537:202:73:163:2 & $<0.001$ \\
\hline Depth of invasion (mucosa) & $10(8.9)$ & $792(81.1)$ & $<0.001$ \\
\hline pN stage (N0:N1:N2:N3) & $76: 26: 7: 3$ & $962: 8: 5: 2$ & $<0.001$ \\
\hline Treatment (endoscopic resection:surgical resection) & $0: 112$ & $540: 437$ & $<0.001$ \\
\hline \multicolumn{4}{|l|}{ Mucosal EGCs $(\mathrm{n}=802)$} \\
\hline No. & 10 & 792 & - \\
\hline Age, yr & $67.4 \pm 10.3$ & $65.8 \pm 11.1$ & 0.655 \\
\hline Male sex & $7(70.0)$ & $550(69.4)$ & 0.635 \\
\hline Country (Japan:Korea) & $1: 9$ & $562: 230$ & $<0.001$ \\
\hline Size, $\mathrm{mm}$ & $30.5(11-40)$ & $16.5(1-145)$ & 0.214 \\
\hline Location (lower:middle:upper) & $6: 3: 1$ & 343:353:96 & 0.565 \\
\hline Cell type (WD:MD:PD:poorly-cohesive*:mucinous) & $1: 7: 2: 0: 0$ & 471:159:45:116:1 & $<0.001$ \\
\hline pN stage (N0:N1:N2:N3) & 10:0:0:0 & 789:2:1:0 & 0.981 \\
\hline Treatment (endoscopic resection:surgical resection) & $0: 10$ & 486:306 & $<0.001$ \\
\hline \multicolumn{4}{|l|}{ Submucosal EGCs $(\mathrm{n}=287)$} \\
\hline No. & 102 & 185 & - \\
\hline Age, yr & $68.5 \pm 9.7$ & $65.2 \pm 11.6$ & 0.013 \\
\hline Male sex & $69(67.6)$ & $137(74.1)$ & 0.274 \\
\hline Country (Japan:Korea) & $84: 18$ & $129: 56$ & 0.024 \\
\hline Size, $\mathrm{mm}$ & $28.9(5-132)$ & $28.9(6-119)$ & 0.241 \\
\hline Location (lower:middle:upper) & $34: 43: 25$ & $47: 92: 46$ & 0.322 \\
\hline Cell type (WD:MD:PD:poorly-cohesive*:mucinous) & $37: 27: 21: 13: 4$ & $66: 43: 28: 47: 1$ & 0.029 \\
\hline pN stage (N0:N1:N2:N3) & $66: 26: 7: 3$ & $173: 6: 4: 2$ & $<0.001$ \\
\hline Treatment (endoscopic resection:surgical resection) & $0: 102$ & $54: 131$ & $<0.001$ \\
\hline
\end{tabular}

Data are presented as mean \pm SD, number (\%), or median (range).

LVI, lymphovascular invasion; EGC, early gastric cancer; WD, well differentiated; MD, moderately differentiated; PD, poorly differentiated. *Poorly cohesive carcinoma includes signet ring cell carcinoma.

most significant factor for LVI. As summarized in Table 2, the presence of LVI in mucosal cancer was related to diagnostic criteria applied in Korea $(\mathrm{p}<0.001)$, cell type $(\mathrm{p}<0.001)$, and treatment $(\mathrm{p}<0.001)$. The rates of LVI were higher in MD $(7 / 166$, $4.2 \%)$ and PD adenocarcinomas $(2 / 47,4.3 \%)$ than in WD adenocarcinomas (1/472, 0.2\%). Of the 802 mucosal EGCs, LVI was not found among the 116 poorly-cohesive carcinomas and one mucinous adenocarcinoma. In logistic regression analysis (Table 4), the presence of LVI was inversely related to diagnostic criteria applied in Japan (OR, 0.329; p=0.017).

\section{LVI in submucosal EGCs}

The presence of LVI in submucosal cancer was related to diagnostic criteria applied in Japan $(\mathrm{p}=0.024)$, being older ( $p=0.013)$, advanced $N$-stage $(p<0.001)$, cell types $(p=0.029)$, and treatment $(p<0.001)$ as summarized in Table 2 . The rates of LVI were lowest in poorly-cohesive carcinomas (13/60, 21.7\%) followed by WD (37/103, 35.9\%), PD (21/49, 42.9\%), MD (27/60, $45.0 \%)$, and mucinous $(4 / 5,80 \%)$ adenocarcinomas. The presence of LVI was positively correlated with diagnostic criteria applied in Japan (OR, 4.000; $\mathrm{p}=0.001)$ and being older (OR, 1.031; $\mathrm{p}=0.045$ ), but was inversely correlated with poorly-cohesive car- 
Table 3. Variables Related to the Presence of Lymphovascular Invasion

\begin{tabular}{|c|c|c|c|c|}
\hline \multirow{2}{*}{ Variable } & \multicolumn{2}{|c|}{ Univariate analysis } & \multicolumn{2}{|c|}{ Multivariate analysis } \\
\hline & OR $(95 \% \mathrm{CI})$ & $\mathrm{p}$-value & OR $(95 \% \mathrm{CI})$ & p-value \\
\hline \multicolumn{5}{|l|}{ Depth of invasion } \\
\hline Mucosa & $0.068(0.032-0.145)$ & $<0.001$ & $0.023(0.012-0.044)$ & $<0.001$ \\
\hline Submucosa & 1 & & 1 & \\
\hline Age & 1.031 (1.004-1.059) & 0.026 & $1.027(1.005-1.050)$ & 0.017 \\
\hline \multicolumn{5}{|l|}{ Country } \\
\hline Japan & $2.413(1.185-4.912)$ & 0.015 & $1.042(0.612-1.775)$ & 0.880 \\
\hline Korea & 1 & & 1 & \\
\hline \multicolumn{5}{|l|}{ Sex } \\
\hline Male & $0.736(0.410-1.323)$ & 0.265 & - & - \\
\hline Female & 1 & & & \\
\hline \multicolumn{5}{|l|}{ Cell type } \\
\hline WD & $0.803(0.323-1.994)$ & 0.636 & - & - \\
\hline MD & $1.625(0.692-3.817)$ & 0.265 & - & - \\
\hline Poorly-cohesive* & $0.300(0.114-1.793)$ & 0.051 & - & - \\
\hline Mucinous & $1.869(0.232-15.080)$ & 0.557 & - & - \\
\hline PD & 1 & & & \\
\hline Size & $0.995(0.982-1.009)$ & 0.494 & - & - \\
\hline \multicolumn{5}{|l|}{ Location } \\
\hline Lower & 1.197 (0.544-2.632) & 0.655 & - & - \\
\hline Middle & $0.745(0.358-1.548)$ & 0.430 & - & - \\
\hline Upper & 1 & & & \\
\hline \multicolumn{5}{|l|}{ pN stage } \\
\hline No & $0.185(0.021-1.649)$ & 0.131 & - & - \\
\hline N1 & $1.639(0.163-16.462)$ & 0.675 & - & - \\
\hline N2 & $0.575(0.047-6.955)$ & 0.663 & - & - \\
\hline N3 & 1 & & & \\
\hline \multicolumn{5}{|l|}{ Treatment } \\
\hline Endoscopic resection & $0.000(0)$ & 0.991 & - & - \\
\hline Surgical resection & 1 & & & \\
\hline
\end{tabular}

OR, odds ratio; CI, confidence interval; WD, well differentiated; MD, moderately differentiated; PD, poorly differentiated.

*Poorly cohesive carcinoma includes signet ring cell carcinoma.

cinoma (OR, 0.341; $\mathrm{p}=0.044)$ in logistic regression analysis (Table 4).

\section{Differences between the countries among the 112 EGC cases with LVI}

Analysis of 112 EGCs with LVI found that the invasion depth $(p<0.001)$ and the cell types $(p<0.001)$ differed significantly between the two countries (Fig. 4). Most of the Korean cases with LVI were MD and PD adenocarcinomas, while most of the Japanese cases were WD adenocarcinoma. Of 85 EGC cases with LVI in Japan, there was only one case of mucosal cancer (WD adenocarcinoma) with LVI. The other 84 Japanese cases of EGC with LVI were all submucosal cancers, which consisted of 36 WD, $18 \mathrm{MD}, 13 \mathrm{PD}, 13$ poorly-cohesive carcinomas (including signet ring cell carcinomas), and four mucinous adenocarcinomas. Of 27 Koreans cases of EGC with LVI, nine were mucosal EGCs (seven MD and two PD adenocarcinomas) and 18 were submucosal EGCs (one WD, nine MD, and eight PD adenocarcinomas).

\section{DISCUSSION}

In this cross-sectional study, depth of invasion was the most significant factor correlated with LVI. Moreover, the characteristics of EGCs with LVI differed between mucosal and submucosal EGCs. For mucosal EGCs, LVI was more frequent in diagnostic criteria applied in Korea, whereas it was more frequent in diagnostic criteria applied in Japan for submucosal EGCs. Other 
Table 4. Lymphovascular Invasion-Related Variables for Mucosal and Submucosal Early Gastric Cancers

\begin{tabular}{|c|c|c|c|c|}
\hline \multirow{2}{*}{ Significant variable } & \multicolumn{2}{|c|}{ Mucosal EGCs (n=802) } & \multicolumn{2}{|c|}{ Submucosal EGCs ( $\mathrm{n}=287)$} \\
\hline & Adjusted OR (95\% CI) & p-value & Adjusted OR (95\% CI) & p-value \\
\hline \multicolumn{5}{|l|}{ Country } \\
\hline Japan & $0.329(0.037-0.908)$ & 0.017 & $4.000(1.777-9.007)$ & 0.001 \\
\hline Korea & 1 & & 1 & \\
\hline \multicolumn{5}{|l|}{ Cell type* } \\
\hline WD & $0.386(0.031-4.787)$ & 0.459 & $0.841(0.313-2.260)$ & 0.731 \\
\hline $\mathrm{MD}$ & $1.880(0.372-9.506)$ & 0.445 & $1.430(0.554-3.691)$ & 0.460 \\
\hline Poorly-cohesive & - & - & $0.341(0.120-0.971)$ & 0.044 \\
\hline Mucinous & - & - & $2.743(0.259-29.015)$ & 0.402 \\
\hline PD & 1 & & 1 & \\
\hline \multicolumn{5}{|l|}{ Treatment } \\
\hline Endoscopic resection & $0.000(0)$ & 0.992 & $0.000(0)$ & 0.997 \\
\hline Surgical resection & 1 & & 1 & \\
\hline Age & - & - & $1.031(1.001-1.062)$ & 0.045 \\
\hline \multicolumn{5}{|l|}{ pN stage } \\
\hline No & - & - & $0.158(0.019-1.353)$ & 0.092 \\
\hline N1 & - & - & $1.531(0.156-15.016)$ & 0.714 \\
\hline N2 & - & - & $0.579(0.048-6.936)$ & 0.667 \\
\hline N3 & & & 1 & \\
\hline
\end{tabular}

EGC, early gastric cancer; OR, odds ratio; CI, confidence interval; WD, well differentiated; MD, moderately differentiated; PD, poorly differentiated. *The findings of poorly cohesive carcinoma and mucinous adenocarcinoma are not shown for mucosal cancers, as none of these cell types was found among the 10 mucosal EGC patients with lymphovascular invasion.

Depth of cancer invasion in EGCs with LVI $(n=112)$

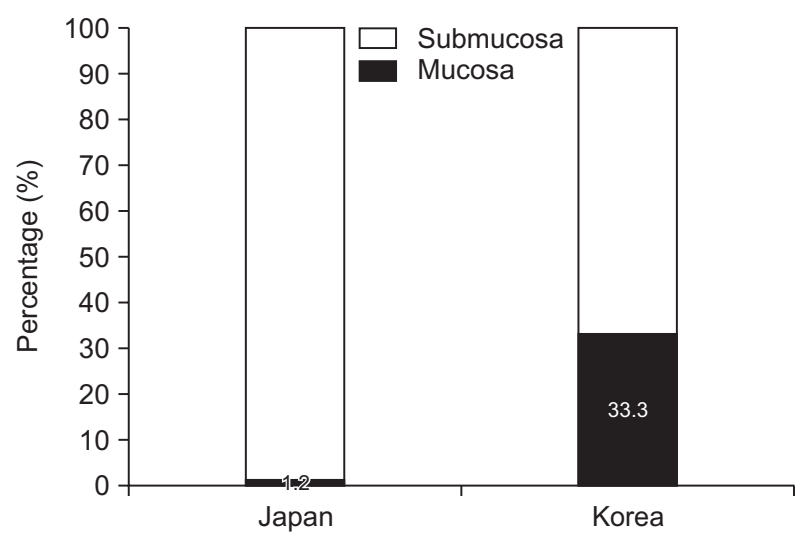

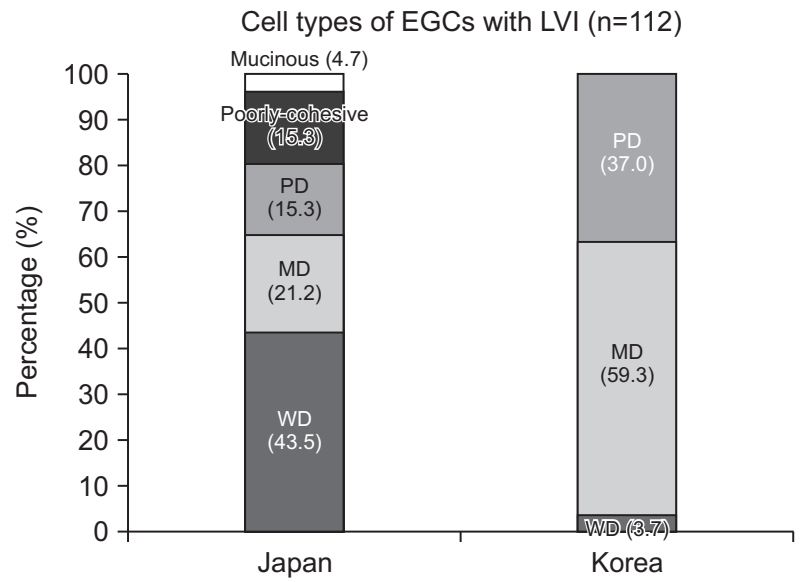

Fig. 4. Findings of the 112 early gastric cancers (EGCs) with lymphovascular invasion (LVI). EGC cases with LVI were found in 85 Japanese cases and 27 Korean cases. There were significant differences between the two countries regarding the depth of invasion $(p<0.001)$ and cell type $(p<0.001)$. $\mathrm{WD}$, well differentiated; MD, moderately differentiated; PD, poorly differentiated.

significant variables for LVI were older age and cell types. Our findings are consistent with those of a recent study showing that all submucosal EGCs need to be checked carefully owing to the high incidence of LVI, regardless of the depth of submucosal invasion. $^{19}$

A particularly notable finding was that the LVI-related variables for EGCs differed significantly between mucosal and submucosal EGCs. Interestingly, the LVI rate was significantly higher in Japan than in Korea for submucosal cancers. Increased detection of LVI in Japanese submucosal EGCs may be due to the different criteria used to determine the final depth of invasion of EGC in the two countries, the higher prevalence of elderly patients in Japan, and the use of different IHC methods. Because the final depth of invasion in Japan includes the loca- 
tion of LVI, while it is the location of cancer cell infiltration in Korea, mucosal EGCs with LVI in Korea are diagnosed as submucosal EGCs in Japan. ${ }^{6-8}$ To minimize the discrepancy in the future, studies on EGCs should show the depth of cancer cell invasion itself and the depth of LVI independently.

Another interesting finding in the present study is that the LVI rate was significantly higher in Korea than in Japan for mucosal cancers. This is because some lesions diagnosed as gastric dysplasia outside of Japan are classified as mucosal adenocarcinoma in Japan like the case shown in Fig. 1. Consequently, the proportion of WD adenocarcinomas was significantly higher in Japan than in Korea in this study. This difference indicates that considerable numbers of intraepithelial adenocarcinomas of the WD type as diagnosed in Japan are diagnosed as dysplasia outside of Japan. Since most of the WD adenocarcinomas did not exhibit LVI in Japanese mucosal cancers, they might have been diagnosed as dysplasia according to the WHO criteria in Korea. Indeed, the EGCs were significantly larger in Korean cases than in the Japanese ones. Our findings could help explain the reported differences in survival rates between Eastern and Western countries after treatment for gastric cancer. ${ }^{3}$ Furthermore, our findings suggest that the Western criteria for dysplasia understates the malignant potential. Together, the higher rate of LVI in Korea for mucosal EGCs indicates that the application of different diagnostic criteria should be considered when interpreting data obtained in different countries.

With regard to cell type, the rate of LVI was lower for poorlycohesive carcinomas and WD adenocarcinomas than for MD, $\mathrm{PD}$, and mucinous adenocarcinomas. This finding is consistent with those of recent studies showing that EGCs with poorlycohesive carcinoma show a low rate of metastasis. ${ }^{20-23}$ In this study, there were significant differences in the composition of EGCs between the two countries. We assume that considerable numbers of cases that are diagnosed as WD adenocarcinoma in Japan are being diagnosed as gastric adenoma with low- or high-grade dysplasia in Korea. Moreover, the higher prevalence of poorly-cohesive carcinoma in Japan suggests that some of these cases would be diagnosed as other types, such as WD or MD adenocarcinomas, in Korea.

In this study, the proportion of endoscopic resection was significantly higher in Japan, because all of the Korean gastric adenomas resected endoscopically were excluded from the beginning. Since endoscopically resected specimens were cut into 2-3 mm width whereas surgical specimens were cut into $1-\mathrm{cm}$ width, we reanalyzed our data after including EGCs that were excluded after endoscopic resection due to LVI (Supplementary Tables 1-4). Nonetheless, LVI was still positively correlated with diagnostic criteria applied in Korea for mucosal EGCs, and LVI was positively correlated with Japanese criteria for submucosal EGCs. Similar to the exclusion of incompletely resected EGCs with LVI, we excluded EGCs with enlarged LN in CT scan that requires $\mathrm{LN}$ dissection. Moreover, metachronous EGCs were also excluded from the beginning, because they exhibit different characteristics such as less invasion and better prognosis than those of primary EGCs. ${ }^{24-26}$ Another reason for excluding metachronous EGCs was the difficulty of proving the origin of LVI or LN metastasis. Sometimes, it is difficult to confirm whether the invasion or metastasis came from the primary or secondary EGC. Therefore, we excluded metachronous and synchronous EGCs to prevent bias.

This study had several limitations. First, different IHC methods were used between the countries. Nevertheless, we strongly suspect that the different LVI rates are mainly due to the different diagnostic criteria rather than IHC method itself based on our previous study. ${ }^{17}$ If the differences were due to other factors than the different diagnostic criteria, LVI rates should be always higher in Japan for both mucosal and submucosal EGCs than those in Korea. Nonetheless, the prevalence of LVI was higher in Japan only for submucosal EGCs, and significantly lower for mucosal EGCs. These contradictory findings suggest that mucosal EGCs in Japan are diagnosed only as adenomas in Korea, and that substantial numbers of submucosal EGCs in Japan are diagnosed as mucosal EGCs in Korea. Second, the depth of cancer cell invasion was not measured as a continuous value (unlike the size of the EGC), and thus could not be expressed in units of milliliters. Moreover, the depth of submucosal invasion was measured in different ways between the surgically and endoscopically resected cases. It was graded into upper third (SM1), middle third (SM2), and lower third (SM3) in surgically resected cases, but was graded into two levels in the endoscopically resected cases: SM1 (penetration into the submucosa less than 500 micrometers from the muscularis mucosa) and SM2 (deeper penetration than SM1). Therefore, we excluded these inconsistent findings from the analysis. Third, the presence of ulcer was not analyzed in this study, because most of the referred cases to our centers exhibited ulcerations due to previous endoscopic biopsies. Despite these limitations, we found significant differences between the two countries with regard to the prevalence of LVI.

In conclusion, the depth of invasion was the most significant factor related to LVI followed by age and cell type. The higher prevalence of LVI in Korea for mucosal EGCs indicates that differences in the diagnostic criteria applied to cell type and depth of invasion between the two countries should be considered when interpreting foreign publications on EGCs. One should be careful in performing endoscopic resection for EGCs based on the good prognosis exhibited in Japanese studies, because EGCs without lamina propria invasion (which is considered as mucosal EGCs in Japan) are already excluded as adenoma or dysplasia in the current WHO classification. In other words, more strict indications for endoscopic resection are required for mucosal EGCs outside Japan, because LVI for mucosal EGCs are higher than those in Japan. Understanding these discrepancies would be helpful for therapeutic endoscopists in improving the 
rate of complete endoscopic resection for EGCs in daily clinical practice.

\section{CONFLICTS OF INTEREST}

No potential conflict of interest relevant to this article was reported.

\section{ACKNOWLEDGEMENTS}

This work was supported by the Korean National Research Foundation (NRF 2016-K2A9A2A-07003625 to Dr. Sun-Young Lee) and the Japan Society for the Promotion of Science (JSPS 16042211-000061 to Dr. Yuji Naito). We thank to Dr. Kiyoshi Ogiso, Dr. Takaaki Murakami, Dr. Yutaka Inada, Dr. Naoto Iwai, and Dr. Tomohiro Ueda at the Department of Molecular Gastroenterology and Hepatology, Kyoto Prefectural University of Medicine for their assistance.

\section{REFERENCES}

1. Schlemper RJ, Kato Y, Stolte M. Review of histological classifications of gastrointestinal epithelial neoplasia: differences in diagnosis of early carcinomas between Japanese and Western pathologists. J Gastroenterol 2001;36:445-456.

2. Sakurai U, Lauwers GY, Vieth M, et al. Gastric high-grade dysplasia can be associated with submucosal invasion: evaluation of its prevalence in a series of 121 endoscopically resected specimens. Am J Surg Pathol 2014;38:1545-1550.

3. Vieth M, Stolte M. Distinction of high-grade intraepithelial neoplasia and tubular gastric adenocarcinoma. In: Kaminishi M, Takubo $\mathrm{K}$, Mafune K, eds. The diversity of gastric carcinoma pathogenesis, diagnosis, and therapy. Tokyo: Springer-Verlag Tokyo, 2005:109116.

4. Schlemper RJ, Riddell RH, Kato Y, et al. The Vienna classification of gastrointestinal epithelial neoplasia. Gut 2000;47:251-255.

5. Stolte M. The new Vienna classification of epithelial neoplasia of the gastrointestinal tract: advantages and disadvantages. Virchows Arch 2003;442:99-106.

6. Lauwers GY, Srivastava A. Gastric preneoplastic lesions and epithelial dysplasia. Gastroenterol Clin North Am 2007;36:813-829.

7. Japanese Gastric Cancer Association. Japanese classification of gastric carcinoma, 14th Japanese edition. 3rd English ed. Tokyo: Kanehara \& Co. Ltd, 2011.

8. Lauwers GY, Carneiro F, Graham DY, et al. Gastric carcinoma. In: Bosman FT, Carneiro F, Hruban RH, Theise ND, eds. WHO classification of tumors of the digestive system. Lyon: International Agency for Research on Cancer, 2010:48-58.

9. Isomoto H, Shikuwa S, Yamaguchi N, et al. Endoscopic submucosal dissection for early gastric cancer: a large-scale feasibility study. Gut 2009;58:331-336.

10. Lee JH, Kim MG, Jung MS, Kwon SJ. Prognostic significance of lymphovascular invasion in node-negative gastric cancer. World J Surg 2015;39:732-739.

11. Kim ER, Lee H, Min BH, et al. Effect of rescue surgery after noncurative endoscopic resection of early gastric cancer. Br J Surg 2015;102:1394-1401.

12. Yamada T, Sugiyama H, Ochi D, et al. Risk factors for submucosal and lymphovascular invasion in gastric cancer looking indicative for endoscopic submucosal dissection. Gastric Cancer 2014;17:692-696.

13. Zheng Z, Zhang Y, Zhang L, et al. A nomogram for predicting the likelihood of lymph node metastasis in early gastric patients. BMC Cancer 2016;16:92.

14. Ahmad R, Setia N, Schmidt BH, et al. Predictors of lymph node metastasis in Western early gastric cancer. J Gastrointest Surg 2016;20:531-538.

15. Wang Z, Ma L, Zhang XM, Zhou ZX. Risk of lymph node metastases from early gastric cancer in relation to depth of invasion: experience in a single institution. Asian Pac J Cancer Prev 2014;15:5371-3575.

16. Kim H, Kim JH, Park JC, Lee YC, Noh SH, Kim H. Lymphovascular invasion is an important predictor of lymph node metastasis in endoscopically resected early gastric cancers. Oncol Rep 2011;25:1589-1595.

17. Kim S, Park HK, Jung HY, et al. ERG Immunohistochemistry as an endothelial marker for assessing lymphovascular invasion. Korean J Pathol 2013;47:355-364.

18. Yonemura Y, Endou Y, Tabachi K, et al. Evaluation of lymphatic invasion in primary gastric cancer by a new monoclonal antibody, D2-40. Hum Pathol 2006;37:1193-1199.

19. Kim JH, Lee YC, Kim H, et al. Additive lymph node dissection may be necessary in minute submucosal cancer of the stomach after endoscopic resection. Ann Surg Oncol 2012;19:779-785.

20. Lee SH, Jee SR, Kim JH, Seol SY. Intramucosal gastric cancer: the rate of lymph node metastasis in signet ring cell carcinoma is as low as that in well-differentiated adenocarcinoma. Eur J Gastroenterol Hepatol 2015;27:170-174.

21. Kim HM, Pak KH, Chung MJ, et al. Early gastric cancer of signet ring cell carcinoma is more amenable to endoscopic treatment than is early gastric cancer of poorly differentiated tubular adenocarcinoma in select tumor conditions. Surg Endosc 2011;25:30873093.

22. Park JM, Kim SW, Nam KW, et al. Is it reasonable to treat early gastric cancer with signet ring cell histology by endoscopic resection? Analysis of factors related to lymph-node metastasis. Eur J Gastroenterol Hepatol 2009;21:1132-1135.

23. Wang Z, Zhang X, Hu J, Zeng W, Zhou Z. Clinicopathological features and outcomes in patients undergoing radical resection for early gastric cancer with signet ring cell histology. J Visc Surg 2015;152:357-361.

24. Kim JW, Jang JY, Chang YW, Kim YH. Clinical features of second primary cancers arising in early gastric cancer patients after endoscopic resection. World J Gastroenterol 2015;21:8358-8365. 
25. Nozaki I, Nasu J, Kubo Y, Tanada M, Nishimura R, Kurita A. Risk factors for metachronous gastric cancer in the remnant stomach after early cancer surgery. World J Surg 2010;34:1548-1554.

26. Gweon TG, Park JM, Lim CH, et al. Increased incidence of second- ary gastric neoplasia in patients with early gastric cancer and coexisting gastric neoplasia at the initial endoscopic evaluation. Eur J Gastroenterol Hepatol 2014;26:1209-1216. 